\title{
ESTADOS DEPRESIVOS Y ATMÓSFERA FAMILIAR EN NIÑOS CON BAJO RENDIMIENTO ESCOLAR
}

\author{
Marío Bulnes Bedón ${ }^{1}$
}

En esta investigación se estudia la depresión infantil en relación con la calidad de vida (en vez de atmósfera familiar) de alumnos de bajo rendimiento escolar en una muestra de 372 escolares de ambos sexos, utilizando el Cuestionario de Depresión para niños de Lang e Ysher; y la Prueba de Calidad de vida de Ohon y Bames, observando en aquellos alumnos un mayor nivel de depresión, mayor afectividad, baja autoestima y dificultad en su interacción social.

Palabras Clave: depresión infantil, calidad de vida, rendimiento escolar.

In this research infant depression is studied in relation with life quality (instead of family atmosphere), in students with low school achievement in a sample of 372 of both genders, using the de Depression Questionnaire for children by Lang e Ysher; and the Test of Life Quality from Olson and Barnes, finding higher levels of depression in the students with more affectivity, low self-esteem and difficulty ill social interaction.

Key words: life's cuality, scholar achievement.

\footnotetext{
1 Miembros de la investigación: César Sarna, Willy Santivañez, Ana Esther Delgado. Colaborador: Jorge Hidalgo.
} 
El presente trabajo trata de estudiar la depresión infantil y la satisfacción dentro de los dominios de las experiencias vitales del niño, como es la vida familiar, amigos, familia extensa, salud, hogar, educación, bienestar económico y comunidad entre otros aspectos; todas estas características dentro del concepto de calidad de vida. Inicialmente se había propuesto también la "Atmósfera familiar", pero se ha creído conveniente ampliar dicho aspecto de un trabajo posterior y de mayor dimensión.

La depresión infantil es un concepto con cierto desacuerdo en cuanto a su presencia o no en los niños, y también desacuerdo sobre las manifestaciones clínicas en función al adulto. Es evidente que en el caso de los niños muchas veces se presenta en forma encubierta, con características de problemas de conducta, de indisciplina, reacciones psiconeuróticas, dificultades del aprendizaje, mal humor, acciones autopunitivas, alteraciones digestivas, del sueño, cefalea y problemas psicomotores, lo que hace difícil su detección.

Son escasas las investigaciones sobre depresión infantil, recién a partir de los años 60 ha aumentado considerablemente el interés y reconocimiento de este fenómeno en la niñez. En el presente trabajo tratamos de buscar el elemento causal de las dificultades del aprendizaje, fobia escolar, apatía, dificultades sociales, baja autoestima y sentimiento de inutilidad. Nuestra finalidad es detectar todos los casos en que se sospeche depresión como factor que interfiere en el aprendizaje escolar. En los problemas de bajo rendimiento escolar también se puede presentar como causa o como efecto la depresión, que podría estar revelando el marco afectivo del escolar, tanto personal y social como familiar.

A su vez, también asumimos que los factores del entorno se hallan fuertemente asociados a los estados emocionales del niño, es por eso que hemos considerado la relación con los demás en términos de "Calidad de vida". El clima psicológico más cercano al niño, que puede ser positivo como un ambiente de cooperación, confianza mutua y respeto, o negativo, en el cual el niño sentirá desconfianza e inseguridad y otras características que tratamos de demostrar tienen que ver con la "Calidad de Vida". 
Por lo tanto, la presente investigación pretende conocer la relación entre depresión infantil, calidad de vida y bajo rendimiento escolar.

\section{PLANTEAMIENTO DEL PROBLEMA}

Todos los cuidados y atenciones que los padres ejercen sobre sus hijos influyen en su desarrollo emocional, y, por lo tanto, en su seguridad personal, base del desarrollo cognoscitivo. Es por eso que se hace necesario establecer el grado de relación social y los estados de ánimo del niño. Sabemos que situaciones difíciles en el medio del niño podrían establecer condiciones emocionales que a su vez interfieren en el aprendizaje; en este caso partimos del estado depresivo del niño y la calidad de vida como factor de interferencia.

Consideramos que al encontrar una relación positiva entre depresión y calidad de vida con respecto al rendimiento escolar, ello posibilitará la intervención precoz a nivel escolar y familiar, en los años básicos de escolaridad, considerando que esta etapa es clave para formular y completar el desarrollo integral del niño.

\section{OBJETIVOS}

Objetivos específicos

1. Establecer la relación entre depresión en el niño de bajo rendimiento escolar y calidad de vida.

2. Comparar el estado depresivo entre niños de bajo y alto rendimiento.

3. Comparar la calidad de vida entre niños de bajo y alto rendimiento.

4. Adecuación del cuestionario de depresión para niños de M. Lang y M.Tisher.

5. Adecuación de la prueba de calidad de vida de David H. Olson y Howard, L.Bames. 


\section{Objetivos generales}

1. Establecer la relación entre depresión en el niño y el bajo rendimiento escolar.

2. Establecer la relación entre calidad de vida y bajo rendimiento escolar.

3. Establecer la relación de calidad de vida y el bajo rendimiento escolar.

\section{HIPÓTESIS}

$\mathrm{H}^{1}$ Existe relación entre depresión y el bajo rendimiento, en la muestra estudiada.

$\mathrm{H}^{2}$ Existe relación entre calidad de vida y el bajo rendimiento escolar en la muestra estudiada.

$\mathrm{H}^{3}$ Existe relación entre calidad de vida y la depresión en niños escolares de bajo rendimiento escolar.

$\mathrm{H}^{4}$ Existe diferencia estadísticamente significativa en los estados depresivos en niños escolares de bajo y alto rendimiento escolar.

$\mathrm{H}^{5}$ Existe diferencia estadísticamente significativa en la calidad de vida en niños escolares de bajo y alto rendimiento escolar.

\section{MARCO TEÓRICO}

\section{Depresión Infantil}

El diagnóstico del síndrome depresivo es mucho más difícil que en la edad adulta; ello se debe, en parte, a que el niño tiene mayor dificultad para describir su sintomatología. En cuanto a la depresión abierta algunos 
autores señalan que los niños pueden mostrar una depresión afectiva, es decir un estado afectivo de desgracia o tristeza crónica. Los niños pueden presentar mal rendimiento y desajuste en la escuela, alteraciones de los patrones de sueño y de la alimentación, sentimiento de miedo y de desesperación, retardo psicomotor, y, en ocasiones, ideas o intentos suicidas. Con frecuencia pueden intercalarse periodos de agitación y ansiedad. En algunos casos puede haber un trauma severo y reciente, relacionado a menudo con la pérdida de un objeto y la presencia de psicopatología grave en familiares cercanos.

Stack (1971; en Lang y Tisher, 1990) clasifica las depresiones infantiles en dos grupos: a) depresiones en niños preescolares, caracterizado por hiperactividad, apatía y somatización, y b) depresiones en niños escolares, caracterizados por depresión simple, fobias u obsesiones, estados depresivos mixtos y depresiones asociadas con disfunciones cerebrales y estados psicóticos.

Podemos considerar como sintomatología de la depresión infantil lo siguiente:

a) Respuesta afectiva: sentimiento de tristeza, desgracia y llantos.

b) Autoconcepto negativo: sentimientos de inadecuación, baja autoestima, inutilidad, desamparo y falta de afecto.

c) Disminución de la productividad mental y de los impulsos: aburrimiento, falta de energía, descontento, baja capacidad para disfrutar el placer y para aceptar ayuda, así como retraso motor.

d) Problemas psicosomáticos: dolores de cabeza y abdominales, perturbaciones del sueño.

e) Preocupaciones: por la muerte, la enfermedad, así como pensamientos suicidas y sentimiento de pérdida, real 0 imaginario.

f) Problemas de agresión: irritabilidad y explosiones de mal humor. 


\section{Calidad de vida}

Una característica común de los estudios de la calidad de vida es la elevación de la satisfacción dentro de los dominios de las experiencias vitales de los individuos. Cada dominio se enfoca sobre una faceta particular de la experiencia vital, como por ejemplo la vida familiar, amigos, familia extensa, domicilio y facilidades de vivienda, educación, empleo, religión, bienestar económico, vecindario y comunidad, salud e impacto de los medios de comunicación masiva. Idealmente tomados en conjunto, estos dominios abarcan la mayor parte de experiencias vitales de la población en general.

Un intento de definir este concepto incluiría las siguientes especificaciones:

a) El termino "vida" se refiere única y exclusivamente a la vida humana en su versión no tanto individual como comunitaria y social. Interesa fundamentalmente a la calidad de vida de amplios agregados sociales.

b) Así mismo el término "vida" quiere hacer referencia a una forma de existencia superior a la meramente física, y que incluiría el ámbito de las relaciones sociales del individuo, sus posibilidades de acceso a los bienes culturales, su entorno ecológico-ambiental, los riesgos a que se encuentra sometida su salud física y psicológica, etc.

c) De modo concreto se puede asimilar el termino "vida" a "actividad", la forma más específicamente humana de relacionarse con el mundo. De esta manera, la calidad de vida se da en muchos casos como sinónimo de la .calidad de las condiciones en que se van desarrollando las diversas actividades del individuo, condiciones a la vez objetivas y subjetivas, cuantitativas y cualitativas.

d) Dicho de otra manera la calidad de vida no es el resultado de una 
aproximación cuantitativa y estricta o mayoritariamente económica a la cuestión de "como vive la gente".

e) El sustantivo "calidad" hace referencia a la naturaleza más o menos satisfactoria de una cosa, o, si se prefiere, a aquellas propiedades inherentes a una cosa que permiten apreciarla como igual, mejor o peor que las restantes de su especie.

f) Al parecer la pieza central de la calidad de vida es la comparación de los atributos o características de una cosa con las que poseen otras del entorno.

g) La comparación que lleva a cabo el individuo la realiza con cargo a criterios de valoración personal, de tal manera que la calidad de vida será considerada como fruto de la percepción individual y subjetiva de unas condiciones de vida objetivas; dicha evaluación se lleva a cabo con cargo a criterios personales (niveles de aspiración, expectativas, grupos de referencia, valores personales, etc.).

h) La calidad de vida se relaciona con el nivel de satisfacción que a uno le proporcionan sus condiciones de vida cuando las compara con la situación en que se desenvuelve la vida de otras personas.

i) La naturaleza de este fenómeno abarca un amplio abanico de materias y disciplinas como son: la Psicología Social, Estudios Ambientales, Sociología, Antropología Cultural, Política, Economía del Bienestar, etc.

\section{PROCEDIMIENTO METODOLÓGICO}

\section{Población y muestra}

El trabajo de investigación a realizar corresponde a una investigación básica, que hará uso del método descriptivo-comparativo. Se aplicará la técnica de observación indirecta a través de cuestionarios. 
La población está constituida por 372 escolares de ambos sexos de colegios tanto estatales como particulares de Lima Metropolitana. El muestreo es de tipo aleatorio y abarca niños de algunos centros educativos, distribuido en forma polarizada en cuartiles superior e inferior, en el primer caso se presenta 91 escolares y en el segundo caso 131 escolares del quinto grado, cuyas edades se encuentran entre los 10 a 12 años de edad. El cuartil medio que asciende a 150 escolares fue descartado para quedamos con los dos grupos extremos o diferenciados, quedando la muestra constituida en 222 escolares. La investigación es de tipo descriptivo, comparativo y correlacional.

La distribución por cuartiles se realizó sobre la base de las notas promedio de su rendimiento escolar, como se puede ver en los cuadros 1,2 Y 3 respectivamente.

\section{Instrumento}

Se utilizó el Cuestionario de Depresión para niños de M.Lang y M. Ysher, el cual procede del Australian Council for Educational Research, adaptado por TEA Ediciones de Madrid. Es una prueba de 66 items, 48 de tipo depresivo y 18 de tipo denominado de tipo positivo. Estos dos conjuntos se mantienen separados y permiten dos subescalas generales independientes: total depresivo y total positivo.

Dentro de cada una de estas dos denominaciones se agruparon sus items por el contenido de los mismos en varias subescalas, que podrían describirse de la siguiente manera:

Total Depresivo: seis sub escalas, 48 items

R.A. Respuesta Afectiva

P.S. Problemas Sociales

A.E. Autoestima

P.M. Preocupación por la muerte/ salud

S.C. Sentimiento de culpabilidad

D.V. Depresivos varios 
Total Positivo: dos sub escalas, 18 items
A.A. Animo - Alegría
P.V. Positivos Varios

También se aplicó la Prueba de Calidad de Vida, de David H. Olson y Howard L. Barnes, el cual consta de 25 items y once dominios específicos, que son los que a continuación se detalla:

\author{
Vida Familiar \\ Amigos \\ Familia Extensiva \\ Salud \\ Hogar \\ Educación \\ Ocio \\ Religión \\ Medios de Comunicación \\ Bienestar Económico \\ Vecindario y Comunidad
}

\title{
PRESENTACIÓN Y ANÁLISIS DE RESULTADOS
}

En la tabla 1 se presentan las medias y la desviación standard, tanto de los escolares del cuartil superior como inferior, con su respectivo análisis comparativo a través del estadístico "t", tomando en cuenta los factores depresivos y la escala de calidad de vida en forma global. A su vez se realiza una ampliación del trabajo presentando una matriz de correlación intra-test e inter-test. 
Tabla 1. Análisis comparativo de los estudiantes del cuartil superior (QSUP / $\left.N^{\circ}=91\right)$ e inferior $\left(Q I N F / N^{\circ}=131\right)$ en el Cuestionario de Depresión y Calidad de Vida.

\begin{tabular}{|c|c|c|c|c|c|}
\hline \multirow[t]{2}{*}{ FACTORES } & \multicolumn{2}{|c|}{ PROMEDIO } & \multicolumn{2}{|c|}{$\begin{array}{l}\text { DESVIACION } \\
\text { ESTÁNDAR }\end{array}$} & \multirow[t]{2}{*}{ "t" } \\
\hline & OSUP & OINF & OSUP & OINF & \\
\hline ANIMO-ALEGRIA & 16.40 & 17.32 & 4.63 & 4.72 & -1.2719 \\
\hline RESP.-AFECTIVA & 17.59 & 20.24 & 6.66 & 6.84 & $-2.5169 *$ \\
\hline PROBS. SOCIALES & 19.23 & 21.77 & 6.84 & 7.43 & $-2.2682 *$ \\
\hline AUTOESTIMA & 18.23 & 22.24 & 7.27 & 7.17 & $-3.5487 *$ \\
\hline $\begin{array}{l}\text { PREOCUPACION } \\
\text { MUERTE-SALUD }\end{array}$ & 16.12 & 19.00 & 5.02 & 5.96 & $-33102 *$ \\
\hline $\begin{array}{l}\text { SENTIMIENTO } \\
\text { DE CULPABIL. }\end{array}$ & 20.93 & 22.80 & 7.03 & 6.58 & -1.7760 \\
\hline $\begin{array}{l}\text { DEPRESIVOS- } \\
\text { VARIOS }\end{array}$ & 28.69 & 29.63 & 4.74 & 5.85 & -1.1219 \\
\hline $\begin{array}{l}\text { POSITIVOS } \\
\text { VARIOS }\end{array}$ & 23.26 & 25.30 & 5.45 & 5.98 & $-2.2794 *$ \\
\hline $\begin{array}{l}\text { TOTAL } \\
\text { DEPRESION }\end{array}$ & 120.84 & 135.71 & 32.61 & 34.11 & $-2.8536^{*}$ \\
\hline $\begin{array}{l}\text { TOTAL } \\
\text { POSITIVO }\end{array}$ & 39.66 & 42.63 & 8.79 & 9.67 & -2.0514 \\
\hline $\begin{array}{l}\text { CALIDAD } \\
\text { DE VIDA }\end{array}$ & 93.90 & 90.20 & 14.54 & 16.00 & 1.5431 \\
\hline
\end{tabular}

* Diferencia significativa al nivel de 0.05

Se puede observar un mayor nivel depresivo en escolares del cuartil inferior (bajo rendimiento escolar) en función a los escolares del cuartil superior, alcanzando un estadístico "t" de -2.8536 , lo cual nos permite determinar que la diferencia es estadísticamente significativa.

Realizando un análisis de los factores que conforman la prueba de depresión y calidad de vida (tabla 2) se puede observar lo siguiente: 
Tabla 2. Correlación entre depresión calidad de vida y rendimiento escolar

\begin{tabular}{|l|l|l|l|l|l|l|l|l|l|l|l|}
\hline & CODo & A1PD & A2PD & A3PD & A4PD & A5PD & A6PD & A7PD & A8PD & A9PD & A10PD \\
\hline & & & & & & & & & & & \\
\hline AA & AIPD & 1.000 & & & & & & & & & \\
\hline R.A. & A2PD & 0.383 & 1.000 & & & & & & & & \\
\hline PROBS & A3PD & 0.356 & 0.850 & 1.000 & & & & & & & \\
\hline AUT & A4PD & 0.390 & 0.761 & 0.803 & 1.000 & & & & & & \\
\hline PRE.S & A5PD & 0.267 & 0.667 & 0.657 & 0.675 & 1.000 & & & & & \\
\hline S.C. & A6PD & 0.251 & 0.615 & 0.634 & 0.675 & 0.587 & 1.000 & & & & \\
\hline D.V. & A7PD & 0.106 & 0.617 & 0.572 & 0.591 & 0.573 & 0.540 & 1.000 & & & \\
\hline P.V, & A8PD & 0.585 & 0.337 & 0.321 & 0.246 & 0.203 & 0.164 & 0.010 & 1.000 & & \\
\hline TD & A9PD & 0.354 & 0.895 & 0.900 & 0.897 & 0.812 & 0.804 & 0.753 & 0.262 & 1.000 & \\
\hline T.Pt. & A10PD & 0.859 & 0.400 & 0.377 & 0.346 & 0.258 & 0.226 & 0.058 & 0.918 & 0.339 & 1.000 \\
\hline C.V. & BIPD & -0.345 & -0.241 & 0.284 & -0.219 & -0.210 & -0.180 & -0.140 & -0.331 & -0.254 & -0.378 \\
\hline NOTA & CI & -0.191 & -0.264 & 0.238 & -0.266 & -0.232 & -0.158 & -0.152 & -0.187 & -0.261 & -0.212 \\
\hline
\end{tabular}

1. Se observa diferencia estadísticamente significativa en el puntaje total de depresión entre los alumnos de bajo rendimiento escolar y alumnos de alto rendimiento escolar ("t" - 2.8536). Los alumnos de bajo rendimiento presentan mayor nivel depresivo.

2. No se observa diferencia estadísticamente significativa en el factor "Calidad de vida" entre los alumnos de bajo rendimiento escolar y alumnos de alto rendimiento escolar ("t" 1.5431).

3. No se observa correlación entre "Calidad de vida" y "Depresión" en alumnos de bajo rendimiento escolar. (O - 0.254).

4. No se observa correlación entre "Depresión" y el bajo rendimiento escolar en la muestra estudiada. 
5. Desde el punto de vista de los factores que integra la prueba de "Depresión" se puede observar lo siguiente:

5.1. Se observa diferencia estadísticamente significativa en:

a) Respuesta afectiva (t: -2.5169), a favor de los alumnos de bajo rendimiento escolar.

b) Problemas sociales (t: -2.2682), denotando dificultades en la interacción social, aislamiento y soledad en el escolar de bajo rendimiento.

c) Autoestima (t: -3.5487), observándose dificultades en los sentimientos, conceptos y actitudes del alumno de bajo rendimiento escolar en relación con su propia estima y valor personal.

d) Preocupación por la Muerte-Salud (t: -3.3102), el cual alude a los sueños y fantasías del alumno de bajo rendimiento escolar en relación a su preocupación por una probable enfermedad.

e) Se puede decir que las características depresivas son más encubiertas, más internas en alumnos de bajo rendimiento escolar, ya que en los aspectos de su relación con los demás "Calidad de vida" no se observa diferencia estadísticamente significativa (t: 1.5431).

5.2. No se observa diferencia estadísticamente significativa en los factores depresivos:

a) Animo-alegría.

b) Sentimiento de culpabilidad.

c) Depresivos varios. 


\section{CONCLUSIONES}

1. Se observa un mayor nivel depresivo en los alumnos de bajo rendimiento escolar.

2. Se observa una respuesta más afectiva en los alumnos de bajo rendimiento escolar

3. Los alumnos de bajo rendimiento escolar presentan una baja autoestima y dificultades en la calidad de su interacci6n social.

4. Existe preocupaci6n por su salud, el cual alude a sueños y fantasías en relaci6n con su enfermedad y muerte

5. Se observa una correlaci6n baja y negativa entre Calidad de vida y depresi6n en la muestra estudiantil.

6. Se observa una correlaci6n baja y negativa entre el rendimiento y el factor de depresi6n.

\section{BIBLIOGRAFÍA}

Annel, A.L. (1972). Estado depresivo en niños y adolescentes. New York:

Halsted Press.

Beck, A.T. (1967). Depression. New York: Harper \& Row.

Kazdin, A. (1996). Tratamiento de la conducta antisocial en la infancia y la adolescencia. Barcelona: Martínez Roca.

Vásquez, G. C. (1985). Trastornos del aprendizaje. Vol. II. Madrid: 
Mario Bulnes 